\title{
Indocyanine green accused
}

\section{T L Jackson}

\section{The case for and against ICG assisted macular surgery}

$\checkmark r$ he concept of retinal vital staining is not new. ${ }^{1-3}$ There has however been renewed interest in the subject since indocyanine green (ICG) was introduced as a vital stain to highlight the internal limiting membrane (ILM) during macular surgery. ICG's well known role in cardiac and choroidal angiography exploits its properties as a fluorophore, but its role as a macular vital stain relies on its properties as a chromophore or biological stain. Biological stains such as ICG have specific atomic groupings that impart colour $(\mathrm{C}=\mathrm{S}, \mathrm{C}=\mathrm{N}, \mathrm{N}=\mathrm{N}, \mathrm{N}=\mathrm{O}$, and $\mathrm{NO}_{2}$ ). ICG is therefore able to stain the thin, optically clear ILM, assisting maculorhexis in much the same way that trypan blue assists capsulorhexis. ICG has more recently been used to help remove epiretinal membranes. ${ }^{4}$

Initial reports were favourable. ${ }^{5-7}$ Subsequently, some of the most prominent exponents of ICG reported possible toxicity, ${ }^{8}$ and enthusiasm waned. More recently there have again been papers showing a favourable outcome, ${ }^{9} 10$ and the debate continues. The article by Hillenkamp et al in this issue of the BJO ( $\mathrm{p} 437$ ) supports the continued use of ICG, but it should not be read in isolation. This editorial presents the case for and against ICG macular vital staining, considering the clinical and experimental evidence in turn.

Hillenkamp's study suggests that ICG vital staining is both safe and effective. It reports a retrospective analysis of two consecutive series of patients undergoing removal of epiretinal membranes, with and without the aid of ICG. The strength of this study lies in its detailed analysis of visual function. Outcome measures included subjective improvement, Amsler grid, corrected visual acuity (VA), slit lamp biomicroscopy, 10 and 30 degree automated perimetry, Goldmann kinetic perimetry, and optical coherence tomography. The results of surgery with and without ICG were broadly similar, except that the ICG group had significantly fewer residual or recurrent epiretinal membranes. As noted by the authors, this may be because of longer follow up in the "no ICG" group, but it is distinctly possible that ICG enhanced membrane removal.
Importantly, this study suggests that visual outcome was not compromised by ICG. This finding agrees with several reports showing good outcome following macular hole surgery with ICG assisted ILM removal. ${ }^{5-7}$

The clinical argument in support of ICG rests not only on reports of good outcome, but also on the deficiencies of the patient studies showing toxicity. Many employed staining routines that are not now thought to be ideal. Some used contact times of several minutes, and solutions of up to $5 \mathrm{mg} / \mathrm{ml}(0.5 \%)$, yet approximately $1 \mathrm{mg} / \mathrm{ml}$ produces adequate staining, ${ }^{10}$ and many surgeons now use exposure times of 30 seconds or less. In addition, many investigators dissolved ICG in $0.5 \mathrm{ml}$ of distilled water, before mixing with $4.5 \mathrm{ml}$ of a balanced saline solution (BSS). This produces a 275 mOsm solution that is hypo-osmotic relative to agents such as BSS (302 mOsm). ${ }^{11}$ It has been suggested that ICG toxicity is caused, ${ }^{12}$ or at least aggravated, ${ }^{11}$ by low osmolarity.

The experimental reports of ICG toxicity are also imperfect. One of the most influential studies exposed human cadaveric eyes to ICG and illumination with a surgical endolight. ${ }^{13}$ The selected histological images were dramatic, with profound disruption of cellular architecture. This is not consistent with clinical observations, as reports of ICG toxicity generally show only mild to moderate deficits. Other authors were not able to repeat these observations in freshly enucleated pig eyes. ${ }^{14}$ Further, the validity of using enucleated eyes to model vascularised, viable retina is uncertain.

\section{And the verdict? A good judge will} want to hear more evidence

Studies of surgical ILM specimens have included retinal cellular elements and this has been marshalled as evidence that ICG alters the cleavage plane during ILM removal. ${ }^{15}$ However, this has also been observed in studies that did not use ICG. ${ }^{16}$ Cell culture experiments also need to be interpreted cautiously, as they do not simulate the complex interactions of a multicellular environment such as the retina. Many authors relied on a mitochondrial dehydrogenase enzyme assay to measure cell viability, yet there is spectral overlap of ICG absorption and the blue formazan reaction product that this assay reads. Without careful rinsing routines or appropriate assay correction, it is possible that residual ICG may produce falsely low readings of viability in a concentration dependent manner. ${ }^{17}$ This may explain why some papers showed more damage with this technique than with ultrastructural analysis. ${ }^{18}$ Potentially useful live animal studies showing histological or electophysiological damage failed to model the clinical use of ICG, with exposure times of several days. ${ }^{19}$

It should be noted that not all experimental studies show that ICG is unsafe. Retinal pigment epithelium $(\mathrm{RPE})^{11} 2021$ and glial ${ }^{11}$ cell culture studies, as well as macular surgery in enucleated pig eyes, ${ }^{14}$ all found that at least some clinically useful preparations did not cause damage.

As in the case supporting ICG use, the case against ICG can be considered in terms of the clinical and experimental evidence. Clinical studies have shown visual field defects, ${ }^{22}$ loss of $\mathrm{VA}^{15}$ and RPE atrophy. ${ }^{23}$ The only randomised trial of ICG vital staining showed a small but significant reduction in VA, albeit using a hypo-osmolar preparation. ${ }^{24}$ Of particular concern are reports showing the persistence of ICG for several weeks. ${ }^{25}$ In addition, the use of ICG may be harder to justify now that $0.15 \%$ trypan blue is available as an alternative chromophore (Membrane Blue, Dorc, Netherlands).

There are experimental studies showing ICG toxicity in $\mathrm{RPE}^{18}$ Müller cells, ${ }^{11}{ }^{13}$ retinal ganglion cells, ${ }^{26}$ and both ex vivo ${ }^{15}$ and in vivo ${ }^{19}$ whole retina. The fact that so many investigators found evidence of cell damage cannot be ignored, even if exposure routines were sometimes dissimilar to those used clinically.

Advocates of ICG may suggest that infracyanine green is a safe alternative but this may not be so. Infracyanine green (Serb, Paris, France) is similar to ICG, but does not contain iodine and comes with 5\% glucose as the diluent. Some investigations suggest that this alters the absorption profile, ${ }^{27}$ reducing the risk of ICG mediated phototoxicity when cells are illuminated. ${ }^{17}$ However, experiments in cadaver eyes also show cell damage, ${ }^{28}$ and Hillenkamp's paper suggests that the effect on the absorption profile may be less than initially thought.

And the verdict? A good judge will want to hear more evidence. What is needed experimentally is a well controlled study that includes vitrectomy, 
short exposure times, then careful histological and electrophysiological analysis-preferably in an animal with rods, cones, and a duplex retinal circulation. Although difficult in some species, surgery would ideally include posterior vitreous detachment and removal of the ILM. Clinically, an appropriately sized, preferably multicentre, randomised trial is needed using an iso-osmotic, low concentration ICG, brief exposure times, and appropriate outcome measures. The parameters measured in Hillenkamp's paper represent a useful template, but they did not include electrophysiology or fluorescein angiography.

Until such trials are completed, clinicians who continue to use ICG have some guidance from the literature in terms of reducing potential risk. One suggestion is to restrict use to difficult cases, where the surgical assistance offered by ICG is likely to outweigh any risk of visual loss. The literature suggests using a low concentration (0.5$1.25 \mathrm{mg} / \mathrm{ml})$, avoiding hypo-osmotic preparations, keeping the endo-illumination distance up and power down, selecting halogen rather than xenon light sources, and rinsing fully and immediately after dye application. Another suggested technique involves the application of viscoelastic over macula holes, to prevent direct contact with the RPE.

Given that the courtroom analogies of this editorial could potentially reflect a medicolegal reality, discussing the use of ICG in the consent process may be worthwhile. Having heard the case for and against ICG, patients can then reach their own verdict.

Br J Ophthalmol 2005;89:395-396.

doi: $10.1136 /$ bjo. 2004.055558

Correspondence to: Timothy L Jackson, Academic Department of Ophthalmology, The Rayne Institute, St Thomas's Hospital, Lambeth
Palace Road, London SE1 7EH, UK; timljackson@hotmail.com

Competing interests: none declared

\section{REFERENCES}

1 Sorsby A, Elkeles A, Goodhart GW, et al. Experimental staining of the retina in life. Proc Roy Soc Med 1937;30:1271-3.

2 Black GW. Some aspects of the treatment of simple detachment of the retina, including vital staining of the retina by methylene blue. Trans Ophthal Soc UK 1947:67:313-22.

3 Kutschera E. [Vital staining of the detached retina with retinal breaks]. Alb Von Graef Archiv Klin Exp Ophthalmol 1969;178:72-87.

4 Foster RE, Petersen MR, Da-Mata AP, et al. Negative indocyanine green staining of epiretinal membranes. Retina 2002;22:106-8.

5 Kadonosono K, Itoh N, Uchio E, et al. Staining of internal limiting membrane in macular hole surgery. Arch Ophthalmol 2000;118:1116-8.

6 Da Mata AP, Burk SB, Riemann CD, et al. Indocyanine green-assisted peeling of the retinal internal limiting membrane during vitrectomy surgery for macular hole repair. Ophthalmology 2001;108:1187-92

7 Kwok AK, Li WW, Pang CP, et al. Indocyanine green staining and removal of internal limiting membrane in macular hole surgery: histology and outcome. Am J Ophthalmol 2001;132:178-83.

8 Gandorfer A, Haritoglou C, Gass CA, et al. Indocyanine green-assisted peeling of the internal limiting membrane may cause retinal damage. Am J Ophthalmol 2001;132:431-3.

9 Slaughter K, Lee IL. Macular hole surgery with and without indocyanine green assistance. Eye 2004; 18:376-8

10 Kwok AK, Lai TY, Yew DT, et al. Internal limiting membrane staining with various concentrations of indocyanine green dye under air in macular surgeries. Am J Ophthalmol 2003;136:223-30.

11 Jackson TL, Hillenkamp J, Knight BC, et al. Safety testing of indocyanine green and trypan blue using retinal pigment epithelium and glial cell cultures. Invest Ophthalmol Vis Sci 2004;45:2778-85.

12 Stalmans P, Van Aken EH, Veckeneer M, et al Toxic effect of indocyanine green on retinal pigment epithelium related to osmotic effects of pigment epithelium related to osmotic effects of

13 Gandorfer A, Haritoglou C, Gandorfer A, et al. Retinal damage from indocyanine green in experimental macular surgery. Invest Ophthalmol Vis Sci 2003;44:316-23.

14 Grisanti S, Szurman P, Gelisken F, et al. Histological findings in experimental macular surgery with indocyanine green. Invest Ophthalmol Vis Sci 2004:45:282-6.

15 Haritoglou C, Gandorfer A, Gass CA, et al. Indocyanine green-assisted peeling of the internal limiting membrane in macular hole surgery affects visual outcome: a clinicopathologic correlation. Am J Ophthalmol 2002;134:836-41.

16 Eckardt C, Eckardt U, Groos S, et al. [Removal of the internal limiting membrane in macular holes. Clinical and morphological findings] Ophthalmologe 1997;94:545-51.

17 Jackson TL, Vote B, Knight BC, et al. Safety testing of infracyanine green using retinal pigment epithelium and glial cell cultures. Invest Ophthalmol Vis Sci 2004;45:3697-703.

18 Sippy BD, Engelbrecht NE, Hubbard GB, et al. Indocyanine green effect on cultured human retinal pigment epithelial cells: implications for macular hole surgery. Am J Ophthalmol 2001;132:433-5.

19 Enaida H, Sakamoto T, Hisatomi T, et al. Morphological and functional damage of the retina caused by intravitreous indocyanine green in rat eyes. Graefes Arch Clin Exp Ophthalmol 2002;240:209-13

20 Ho JD, Tsai RJ, Chen SN, et al. Cytotoxicity of indocyanine green on retinal pigment epithelium: implications for macular hole surgery. Arch Ophthalmol 2003;121:1423-9.

21 Gale JS, Proulx AA, Gonder JR, et al. Comparison of the in vitro toxicity of indocyanine green to that of trypan blue in human retinal pigment epithelium cell cultures. Am J Ophthalmol 2004; 138:64-9

22 Uemura A, Kanda S, Sakamoto Y, et al. Visual field defects after uneventful vitrectomy for epiretinal membrane with indocyanine greenassisted internal limiting membrane peeling. Am J Ophthalmol 2003;136:252-7.

23 Engelbrecht NE, Freeman J, Sternberg Jr P, et al. Retinal pigment epithelial changes after macular hole surgery with indocyanine green-assisted internal limiting membrane peeling. Am J Ophthalmol 2002;133:94.

24 Horio N, Horiguchi M. Effect on visual outcome after macular hole surgery when staining the internal limiting membrane with indocyanine green dye. Arch Ophthalmol 2004;122:992-6.

25 Weinberger AWA, Kirchhof B, Mazinani BE,

et al. Persistent indocyanine green (ICG)

fluorescence 6 weeks after intraocular ICG administration for macular hole surgery. Graefes Arch Clin Exp Ophthalmol 2001;239:388-90.

26 Iriyama A, Uchida S, Yanagi Y, et al. Effects of indocyanine green on retinal ganglion cells. Invest Ophthalmol Vis Sci 2004;45:943-7.

27 Haritoglou C, Gandorfer A, Schaumberger M, et al. Light-absorbing properties and osmolarity of indocyanine-green depending on concentration and solvent medium. Invest Ophthalmol Vis Sci 2003;44:2722-9.

28 Haritoglou C, Gandorfer A, Gass CA, et al. Histology of the vitreoretinal interface after staining of the internal limiting membrane using glucose $5 \%$ diluted indocyanine green and infracyanine green. Am J Ophthalmol 2004; 137:345-8.

\section{Ex-PRESS implant}

\section{A Mermoud}

\section{Fast, simple, safe, efficient?}

$\mathrm{n}$ the past 5 years, the Ex-PRESS drainage device has been mentioned in the discussions of glaucoma surgeons. Its place in the surgical management of glaucoma has not been clear so far and much contradictory information and controversy have been circulated.
In this issue of the BJO ( $\mathrm{p} 425)$ is presented the first written clinical report on the Ex-PRESS tube used in combined cataract and glaucoma surgery.

Glaucoma drainage devices (GDDs) were first introduced a century ago. Much experience has been gained in the use of these devices, and has led to many modifications in design, construction, and implantation techniques. ${ }^{1}$ In 1969 Molteno launched the concept of a tube and plate for glaucoma drainage, in which the plate is secured onto the episclera, helping with the formation of a posterior orbital filtering bleb. $^{23}$ Contemporary GDDs, which dominate the market are the Molteno, Krupin, Baerveldt, Ahmed, and OptiMed devices, which all share the essential design concept of posterior filtration via a tube (outer diameter $0.56-0.63 \mathrm{~mm}$ ) from the anterior chamber to a plate element secured to the episclera; they differ in plate design and size $\left(135-500 \mathrm{~mm}^{2}\right)$ and their provision for a flow control mechanism. 
If glaucoma drainage devices do offer substantial surgical possibilities for refractory glaucoma they also present disadvantages, either overflow of aqueous onto the anterior chamber or poor filtration with IOP elevation.

Contact between the tube and the corneal endothelium may also induce a long term corneal decompensation. ${ }^{4}$ To minimise the rate of early postoperative hypotony, some of the devices contain a valve mechanism that restricts the aqueous outflow. ${ }^{5-7}$ However, owing to the relatively high postoperative complication rates and the relatively difficult and traumatic implantation procedure, the use of GDDs is usually limited to complicated cases after other modes of treatment, both surgical and nonsurgical, have failed. ${ }^{8}$

Past experience with GDDs reveals that material composition, implant design, physicochemical surface properties, condition of the host bed, surgical technique, and mechanical properties influence the eye's response to the device. All of these factors may influence the overall performance and surgical outcome. ${ }^{910}$

The Ex-PRESS implant is a miniature unvalved glaucoma implant. It was developed as an alternative procedure to trabeculectomy and to the other types of glaucoma filtering surgery for patients with primary open angle glaucoma (POAG). The device is a $3 \mathrm{~mm}$ long stainless steel tube (outer diameter $400 \mu \mathrm{m}$ (27 gauge)) with a bevelled, sharpened, rounded tip, a disc-like flange $\left(<1 \mathrm{~mm}^{2}\right)$ at the device proximal end, and a spur-like projection that prevents its extrusion. The external flange and inner spur are angled to conform to the anatomy of the sclera, and the distance between them corresponds to the scleral thickness at the site of implantation. ${ }^{11}$

Originally, the device was designed to be inserted at the limbus directly under the conjunctiva. In this case, a subconjunctival bleb is formed and serves as a flow modulator. This technique is relatively simple and can be performed in a few minutes, either on its own or in combination with phacoemulsification.

The paper by Traverso et al in this issue of the journal summarises a 3 year follow up with the device. Success rate and short term complications, such as a shallow or flat anterior chamber and hyphaema compare favourably with data reported in the literature for trabeculectomy.

However, some physicians encountered poor conjunctival covering of the device. Several conjunctival erosions were noted over the external flange. Conjunctivoplasty or tube removal had to be performed to avoid secondary infection. On the other hand, several patients showed conjunctival scarring with subsequent decreased aqueous humour filtration.

To overcome this complication of conjunctival erosion and bleb fibroses, Dahan and Carmichel recently suggested implanting the device under a limbus based 50\% deep scleral flap extending into clear cornea. This operation is similar to standard trabeculectomy without the need of an iridectomy or scleral removal. ${ }^{12}$ This may offer an advantage over standard trabeculectomy. Early findings from Sherwood on rabbits (Ex-PRESS filtering shunt bleb TGF- $\beta$ levels in rabbit model, personal communication, October 2004) reveal that the "less invasive technique reduces ocular inflammation by decreasing TGF- $\beta$ level in aqueous humour." The rationale behind this surgical modification is to increase the resistance to aqueous flow in the early postoperative stages as well as to reduce the development of late conjunctival erosion. This technique therefore potentially reduces the complications of hypotony, especially in patients at high risk of surgical failure. ${ }^{12}$ Furthermore, the 2 year postoperative reduction in IOP in the study of Dahan et al was $>40 \%$ and only $10 \%$ of the patients required further medical therapy. ${ }^{12}$

This tube may also be used in deep sclerectomy to simplify the difficult dissection of Schlemm's canal and trabeculo-Descemet's membrane. In an unpublished study, we found that by performing a partial posterior deep sclerectomy and inserting an Ex-PRESS implant into the anterior portion under the superficial scleral flap, we obtained excellent IOP reduction with very few postoperative complications. This technique drastically simplifies the surgical dessection of deep sclerectomy and yet still provides its outflow advantage including the drainage of the aqueous to (1) a subconjunctival bleb, (2) an intrascleral bleb, and (3) the subchoroidal space.
Used in deep sclerectomy, the ExPRESS tube also avoids the late need for goniopuncture (Mermoud et al, unpublished data).

Initial doubt about the Ex-PRESS implant are decreasing with recent advances offering possibly a wider spectrum of indications while diminishing the potential complications.

The Ex-PRESS implant was introduced to offer a quick and simple alternative to glaucoma surgery. The procedure is indeed rapid; however, it may be associated with new complications such as conjunctival erosion and risk of subsequent infection. The modification in the technique by introducing the tube under a superficial scleral flap seems to overcome these complications.

Further research and clinical studies will allow us to discover whether the Ex-PRESS tube will definitely change our approach to glaucoma surgery.

Br J Ophthalmol 2005;89:396-397.

doi: 10.1136/bjo.2004.061689

Correspondence to: A Mermoud, Hopital Ophtalmique Jules Gonin, Avenue de France 15, CH-1004 Lausanne, Switzerland; andre.mermoud@ophtal.vd.ch

\section{REFERENCES}

1 Lim KS, Allan BDS, Lloyed AW, et al. Glaucoma drainage devices; past, present, and future. Br J Ophthalmol 1998;82:1083-9.

2 Molteno ACB. New implant for drainage of glaucoma. Br J Ophthalmol 1969;53:161-8.

3 Schocket SS, Lakhanpal V, Richards RD. Anterior chamber tube shunt to an encircling band in the treatment of neovascular glaucoma. Ophthalmology 1982;89:1188-94

4 Freedman J. Management of the Molteno silicone tube in corneal transplant surgery. Ophthalmic Surg Lasers 1998;29:432-4.

5 Krupin T, Podos SM, Becker B, et al. Valve implants in filtering surgery. Am J Ophthalmol 1976;81:232-5

6 Coleman AL, Hill R, Wilson MR, et al. Initial clinical experience with the Ahmed glaucoma valve implant. Am J Ophthalmol 1995; 120:23-31.

7 Kim DD, Menmen JE. Spontaneous disengagement of the OptiMed implant. Arch Ophthalmol 1996;114:1420-1.

8 Melamed S. Molteno implant surgery in refractory glaucoma. Surv Ophthalmol 1990;34:441-8.

9 Rosengren A, Danielsen N, Bjursten LM. Inflammatory reaction dependence on implant location in the rat soft tissue. Biomaterials 1997:18:979-87.

10 Ayyala RS, Harman LE, Michelini-Norris B, et al. Comparison of different biomaterials for glaucoma drainage devices. Arch Ophthalmol 1999: 117:233-6.

11 Nyska A, Glovinsky Y, Belkin M, et al. Biocompatibility of the Ex-PRESS miniature glaucoma drainage implant. J Glaucoma 2003; 12:275-80.

12 Dahan E, Carmichael TR. Implantation of a miniature glaucoma device under a scleral flap. J Glaucoma 2005; (in press). 
CATARACT SURGERY

\section{Is one trial enough?}

\section{R Wormald}

\section{Is this sufficient evidence to change practice?}

n the March issue of $B J O^{1}$ a rando-

mised controlled trial was reported in which topical versus sub-Tenon's local anaesthesia for routine cataract surgery is compared. The trial is well designed and simple, using a validated outcome measure with the outcome observers masked to the intervention. It shows a clear preference for subTenon's block by patients undergoing routine cataract surgery.

Does this prove it? Is this sufficient evidence to change practice? In making such a decision, readers will want to critically appraise this evidence. Those who disagree with its findings will try hard to find flaws in the study while those that agree with it will tend to take it as face value and feel vindicated. Both sides will have to consider the probabilities that the results of the study could be erroneous or point to the truth.

What kinds of errors occur in trials? After bias and confounding have been dealt with (and by design, these seem to have been well controlled), what about the possibility that chance alone could have produced the result? The $\mathrm{p}$ values are "significant" and the confidence limits of the estimate of effect do not include unity so that by definition, it is improbable that the estimate of effectiveness could have occurred by chance (though they still could have).

The other sort of error, failing to find an effect when in fact there was one, would not seem to be relevant since an effect was shown. The key to this question is the power of the study based on the number of people randomised and the size of effect worth detecting. Discerning readers will note that there is no such power statement in this report.
When I peer reviewed it, I spotted this omission and asked whether the reason for this was that the study was in fact a pilot study. It is now clearly stated in the methods that this study was designed as a pilot since there was no a priori estimate of effect to guide the sample size estimation (this was not stated in the original version).

This is an important point; the study is small and small studies are prone to both kind of errors-alpha type 1 , showing an effect when it is actually just chance, and beta type 2, failing to find an effect when there is one. The weakness in this trial is its small size and its potential for both kinds of errors because chance plays a greater part in smaller experiments. The purpose of a pilot study, among other things, is to get a feeling for a likely effect size and to refine the methods of capturing reliable outcome data. Finding an effect in a pilot study is not a reason not to conduct the definitive experiment. The trialists may need to reappraise the study design and the size of effect that is clinically relevant, then perhaps apply more stringent criteria for the control of an alpha error. It will be the duty of the research ethics committees to make a decision about continuing to withhold the treatment found to be advantageous in the pilot study.

This study, despite being small, is of value and it is right that it should be published, since all results from properly conducted randomised trials add to the body of evidence (even if only in a small way) and should be available in the public domain for all to see. This may soon become a statutory requirement in the United States if legislation to prospectively register all randomised controlled trials, and make their results available to all, becomes law. ${ }^{2}$

This will be a major step forward for evidence based medicine since it will lock the pharmaceutical industry, as well as everybody else, into making the results of all their trials, whether positive or negative in outcome, publicly available.

The body of evidence grows as experiments are repeated. Another properly powered trial with a sample size calculation and with similar findings has been published in the British Journal of Anaesthesia ${ }^{3}$ since Rüschen et al's paper was accepted. These studies should now be summarised in a properly conducted systematic review. One such review has been published and would appear to support the findings of this study. ${ }^{4}$ Another Cochrane review is under way which will include the findings of this trial and the other recently published one. Surgeons wanting to make decisions about changing their practice may decide there is sufficient evidence to do so but others may wish to await a larger body of evidence. It may be difficult to justify another trial comparing subTenon's with topical anaesthesia alone but some will argue that intracameral lignocaine would make a major difference to the comparison. Thus, further trials are needed to address this question.

Br J Ophthalmol 2005;89:398. doi: 10.1136/bjo.2004.055517

Correspondence to: Richard P Wormald, Moorfields Eye Hospital, 162 City Road, London ECIV 2PD, UK; r.wormald@ud.ac.uk

\section{REFERENCES}

1 Rüschen H, Celaschi D, Bunce C, et al. Randomised controlled trial of sub-Tenon's block versus topical anaesthesia for cataract surgery: a comparison of patient satisfaction. $\mathrm{Br} J$ Ophthalmol 2005;89:291-3

2 Roehr R. News round up. BMJ 2004;329:996.

3 Srinivasan S, Fern Al, Selvaraj S, et al. Randomized double-blind clinical trial comparing topical and sub-Tenon's anaesthesia in routine cataract surgery. $\mathrm{Br} J$ Anaesth 2004;93:683-6.

4 Friedman DS, Bass EB, Lubomski LH, et al. Synthesis of the literature on the effectiveness of regional anesthesia for cataract surgery. Ophthalmology 2001; 108:519-29. 